ANNALES

POLONICI MATHEMATICI

$85.2(2005)$

\title{
Parabolic initial-boundary value problems in Orlicz spaces
}

\author{
by A. Elmahi and D. Meskine (Fès)
}

\begin{abstract}
We prove some time mollification properties and imbedding results in inhomogeneous Orlicz-Sobolev spaces which allow us to solve a second order parabolic equation in Orlicz spaces.
\end{abstract}

1. Introduction. Let $\Omega$ be a bounded open subset of $\mathbb{R}^{N}$ and let $Q$ be the cylinder $\Omega \times(0, T)$ with some given $T>0$. In this paper we deal with the following parabolic initial-boundary value problem:

$$
\begin{cases}\partial u / \partial t+A(u)=f & \text { in } Q, \\ u(x, t)=0 & \text { on } \partial \Omega \times(0, T), \\ u(x, 0)=u_{0}(x) & \text { in } \Omega,\end{cases}
$$

where $A$ is an elliptic second order operator of divergence form

$$
A(u)=-\operatorname{div}(a(x, t, u, \nabla u))+a_{0}(x, t, u, \nabla u)
$$

with the coefficients $a$ and $a_{0}$ satisfying the classical Leray-Lions conditions.

Consider, first, the case where $a$ and $a_{0}$ have polynomial growth with respect to $u$ and $\nabla u$. Then $A$ is a bounded operator from $L^{p}\left(0, T ; W_{0}^{1, p}(\Omega)\right)$, $1<p<\infty$, into its dual. In this setting, problems of the form (1) were solved by J.-L. Lions [16] and Brézis-Browder [5] in the case where $p \geq 2$ and by Landes [14] and Landes-Mustonen [15] when $1<p<2$. See also [3] and [4] for related topics.

In the case where $a$ and $a_{0}$ satisfy a more general growth condition with respect to $u$ and $\nabla u$, it is shown in [6] that the appropriate space in which (1) can be studied is the inhomogeneous Orlicz-Sobolev space $W^{1, x} L_{M}(Q)$, where the N-function $M$ is related to the actual growth of $a$ and $a_{0}$. The solvability of (1) in this setting is proved by Donaldson [6] and Robert [18] by assuming that $A$ is monotone, $t^{2} \ll M(t)$ and $\bar{M}$ satisfies a $\Delta_{2}$ condition, and by Elmahi [7] when $M$ satisfies a $\Delta^{\prime}$ condition and $M(t) \ll t^{N /(N-1)}$.

2000 Mathematics Subject Classification: 35K15, 35K20, 35K60.

Key words and phrases: inhomogeneous Orlicz-Sobolev spaces, parabolic problems, mollification. 
It is our purpose in this paper to prove the existence of solutions for problem (1) in the setting of Orlicz spaces without assuming any growth restriction on $M$. We use a Galerkin method due to Landes and Mustonen $[14],[15]$.

Note that, as in [14], we can include a perturbation term of the form $g(x, t, u)$ without difficulties, but we prefer to do this, with a more general term $g(x, t, u, \nabla u)$ having natural growth with respect to $|\nabla u|$, in [8] by using some compactness results.

\section{Preliminaries}

2.1. Let $M: \mathbb{R}^{+} \rightarrow \mathbb{R}^{+}$be an N-function, i.e. $M$ is continuous, convex, $M(t)>0$ for $t>0, M(t) / t \rightarrow 0$ as $t \rightarrow 0$ and $M(t) / t \rightarrow \infty$ as $t \rightarrow \infty$.

Equivalently, $M$ admits the representation $M(t)=\int_{0}^{t} m(\tau) d \tau$, where $m: \mathbb{R}^{+} \rightarrow \mathbb{R}^{+}$is non-decreasing, right continuous, with $m(0)=0, m(t)>0$ for $t>0$ and $m(t) \rightarrow \infty$ as $t \rightarrow \infty$.

The N-function $\bar{M}$ conjugate to $M$ is defined by $\bar{M}(t)=\int_{0}^{t} \bar{m}(\tau) d \tau$, where $\bar{m}: \mathbb{R}^{+} \rightarrow \mathbb{R}^{+}$is given by $\bar{m}(t)=\sup \{s: m(s) \leq t\}$ (see [1], [12] and [13]).

We will extend these $\mathrm{N}$-functions to even functions on all $\mathbb{R}$.

The N-function $M$ is said to satisfy the $\Delta_{2}$ condition if, for some $k>0$,

$$
M(2 t) \leq k M(t) \quad \forall t \geq 0 .
$$

When (3) holds only for $t \geq$ (some) $t_{0}>0$ then $M$ is said to satisfy the $\Delta_{2}$ condition near infinity.

2.2. Let $\Omega$ be an open subset of $\mathbb{R}^{N}$. The Orlicz class $\mathcal{L}_{M}(\Omega)$ (resp. the Orlicz space $L_{M}(\Omega)$ ) is defined as the set of (equivalence classes of) real-valued measurable functions $u$ on $\Omega$ such that $\int_{\Omega} M(u(x)) d x<\infty$ (resp. $\int_{\Omega} M(u(x) / \lambda) d x<\infty$ for some $\lambda>0$ ).

$L_{M}(\Omega)$ is a Banach space under the norm

$$
\|u\|_{M, \Omega}=\inf \left\{\lambda>0: \int_{\Omega} M(u(x) / \lambda) d x \leq 1\right\}
$$

and $\mathcal{L}_{M}(\Omega)$ is a convex subset of $L_{M}(\Omega)$.

The closure in $L_{M}(\Omega)$ of the set of bounded measurable functions with compact supports in $\bar{\Omega}$ is denoted by $E_{M}(\Omega)$. The equality $E_{M}(\Omega)=$ $L_{M}(\Omega)$ holds if and only if $M$ satisfies the $\Delta_{2}$ condition, for all $t$ or for $t$ large according to whether $\Omega$ has infinite measure or not.

The dual space of $E_{M}(\Omega)$ can be identified with $L_{\bar{M}}(\Omega)$ by means of the pairing $\int_{\Omega} u(x) v(x) d x$, and the dual norm on $L_{\bar{M}}(\Omega)$ is equivalent to $\|\cdot\|_{\bar{M}, \Omega}$. 
The space $L_{M}(\Omega)$ is reflexive if and only if $M$ and $\bar{M}$ satisfy the $\Delta_{2}$ condition, for all $t$ or for $t$ large, according to whether $\Omega$ has infinite measure or not.

Two N-functions $M$ and $P$ are said to be equivalent (resp. equivalent near infinity) if there exist real numbers $k_{1}, k_{2}>0$ such that

$$
\left.P\left(k_{1} t\right) \leq M(t) \leq P\left(k_{2} t\right) \quad \text { for all } t \geq 0 \quad \text { (resp. for } t \geq t_{0}>0\right) .
$$

$P \ll M$ denotes that $P$ grows essentially less rapidly than $M$, meaning that $P(\varepsilon t) / M(t) \rightarrow 0$ as $t \rightarrow \infty$, for each $\varepsilon>0$. This is the case if and only if

$$
M^{-1}(t) / P^{-1}(t) \rightarrow 0 \quad \text { as } t \rightarrow \infty,
$$

therefore, we have the continuous imbedding $L_{M}(\Omega) \subset E_{P}(\Omega)$ when $\Omega$ has finite measure.

2.3. We now turn to the Orlicz-Sobolev spaces. $W^{1} L_{M}(\Omega)$ (resp. $\left.W^{1} E_{M}(\Omega)\right)$ is the space of all functions $u$ such that $u$ and its distributional derivatives of order 1 lie in $L_{M}(\Omega)$ (resp. $E_{M}(\Omega)$ ). It is a Banach space under the norm

$$
\|u\|_{1, M, \Omega}=\sum_{|\alpha| \leq 1}\left\|D^{\alpha} u\right\|_{M, \Omega}
$$

Thus $W^{1} L_{M}(\Omega)$ and $W^{1} E_{M}(\Omega)$ can be identified with subspaces of the product of $N+1$ copies of $L_{M}(\Omega)$. Denoting this product by $\prod L_{M}$, we will use the weak topologies $\sigma\left(\prod L_{M}, \prod E_{\bar{M}}\right)$ and $\sigma\left(\prod L_{M}, \prod L_{\bar{M}}\right)$.

The space $W_{0}^{1} E_{M}(\Omega)$ is defined as the (norm) closure of the Schwartz space $\mathcal{D}(\Omega)$ in $W^{1} E_{M}(\Omega)$, and the space $W_{0}^{1} L_{M}(\Omega)$ as the $\sigma\left(\prod L_{M}, \prod E_{\bar{M}}\right)$ closure of $\mathcal{D}(\Omega)$ in $W^{1} L_{M}(\Omega)$.

We say that $u_{n}$ converges to $u$ for the modular convergence in $W^{1} L_{M}(\Omega)$ if for some $\lambda>0, \int_{\Omega} M\left(\left(D^{\alpha} u_{n}-D^{\alpha} u\right) / \lambda\right) d x \rightarrow 0$ for all $|\alpha| \leq 1$. This implies convergence with respect to $\sigma\left(\prod L_{M}, \prod L_{\bar{M}}\right)$.

If $M$ satisfies the $\Delta_{2}$ condition on $\mathbb{R}^{+}$(near infinity only when $\Omega$ has finite measure), then modular convergence coincides with norm convergence.

2.4. Let $W^{-1} L_{\bar{M}}(\Omega)$ (resp. $W^{-1} E_{\bar{M}}(\Omega)$ ) denote the space of distributions on $\Omega$ which can be written as sums of derivatives of order $\leq 1$ of functions in $L_{\bar{M}}(\Omega)$ (resp. $\left.E_{\bar{M}}(\Omega)\right)$. It is a Banach space under the usual quotient norm.

Recall that $\Omega$ is said to have the segment property if there exist an open covering $\left\{U_{i}\right\}$ of $\bar{\Omega}$ and corresponding vectors $\left\{y_{i} \in \mathbb{R}^{N}\right\}$ such that, for $x \in \bar{\Omega} \cap U_{i}$ and $0<t<1, x-t y_{i} \in \Omega$. It was proved in [10] that if the open set $\Omega$ has the segment property, then the space $\mathcal{D}(\Omega)$ is dense in $W_{0}^{1} L_{M}(\Omega)$ for the modular convergence and thus for the topology $\sigma\left(\prod L_{M}, \prod L_{\bar{M}}\right)$. Consequently, the action of a distribution in $W^{-1} L_{\bar{M}}(\Omega)$ on an element of $W_{0}^{1} L_{M}(\Omega)$ is well defined. 
2.5. Let $\Omega$ be a bounded open subset of $\mathbb{R}^{N}, T>0$ and $Q=\Omega \times(0, T)$. Let $M$ be an N-function. For each $\alpha \in \mathbb{N}^{N}$, denote by $D_{x}^{\alpha}$ the distributional derivative on $Q$ of order $\alpha$ with respect to the variable $x \in \mathbb{R}^{N}$. The inhomogeneous Orlicz-Sobolev spaces of order 1 are defined as follows:

$$
W^{1, x} L_{M}(Q)=\left\{u \in L_{M}(Q): D_{x}^{\alpha} u \in L_{M}(Q), \forall|\alpha| \leq 1\right\}
$$

and

$$
W^{1, x} E_{M}(Q)=\left\{u \in E_{M}(Q): D_{x}^{\alpha} u \in E_{M}(Q), \forall|\alpha| \leq 1\right\} .
$$

The latter space is a subspace of the former. Both are Banach spaces under the norm

$$
\|u\|=\sum_{|\alpha| \leq 1}\left\|D_{x}^{\alpha} u\right\|_{M, Q}
$$

We can easily show that they form a complementary system when $\Omega$ satisfies the segment property. These spaces are considered as subspaces of the product space $\prod L_{M}(Q)$ which has $N+1$ factors. We shall also consider the weak topologies $\sigma\left(\prod L_{M}, \prod E_{\bar{M}}\right)$ and $\sigma\left(\prod L_{M}, \prod L_{\bar{M}}\right)$. If $u \in W^{1, x} L_{M}(Q)$ then the function $t \mapsto u(t)=u(\cdot, t)$ is defined on $(0, T)$ with values in $W^{1} L_{M}(\Omega)$. If, further, $u \in W^{1, x} E_{M}(Q)$ then $u(\cdot, t)$ is $W^{1} E_{M}(\Omega)$-valued and is strongly measurable. Furthermore, we have the continuous imbed$\operatorname{ding} W^{1, x} E_{M}(Q) \subset L^{1}\left([0, T], W^{1} E_{M}(\Omega)\right)$. The space $W^{1, x} L_{M}(Q)$ is not in general separable and if $u \in W^{1, x} L_{M}(Q)$, we cannot conclude that $u(t)$ is measurable from $(0, T)$ into $W^{1} L_{M}(\Omega)$. However, the scalar function $t \mapsto\left\|D_{x}^{\alpha} u(t)\right\|_{M, \Omega}$ is in $L^{1}(0, T)$ for all $|\alpha| \leq 1$.

2.6. The space $W_{0}^{1, x} E_{M}(Q)$ is defined as the (norm) closure of $\mathcal{D}(Q)$ in $W^{1, x} E_{M}(Q)$. We can easily show as in [10] that, when $\Omega$ has the segment property, each element $u$ of the closure of $\mathcal{D}(Q)$ with respect to the weak $*$ topology $\sigma\left(\prod L_{M}, \prod E_{\bar{M}}\right)$ is a limit, in $W^{1, x} L_{M}(Q)$, of some sequence $\left(u_{n}\right) \subset$ $\mathcal{D}(Q)$ for the modular convergence, i.e. there exists $\lambda>0$ such that, for all $|\alpha| \leq 1, \int_{Q} M\left(\left(D_{x}^{\alpha} u_{n}-D_{x}^{\alpha} u\right) / \lambda\right) d x d t \rightarrow 0$ as $n \rightarrow \infty$. This implies that $\left(u_{n}\right)$ converges to $u$ in $W^{1, x} L_{M}(Q)$ for the weak topology $\sigma\left(\prod L_{M}, \prod L_{\bar{M}}\right)$. Consequently,

$$
\overline{\mathcal{D}}(Q)^{\sigma\left(\prod L_{M}, \Pi E_{\bar{M}}\right)}=\overline{\mathcal{D}}(Q)^{\sigma\left(\prod L_{M}, \Pi L_{\bar{M}}\right)},
$$

and this space will be denoted by $W_{0}^{1, x} L_{M}(Q)$. Furthermore, $W_{0}^{1, x} E_{M}(Q)=$ $W_{0}^{1, x} L_{M}(Q) \cap \prod E_{M}$.

Poincaré's inequality also holds in $W_{0}^{1, x} L_{M}(Q)$ and there is a constant $C>0$ such that for all $u \in W_{0}^{1, x} L_{M}(Q)$ one has

$$
\sum_{|\alpha| \leq 1}\left\|D_{x}^{\alpha} u\right\|_{M, Q} \leq C \sum_{|\alpha|=1}\left\|D_{x}^{\alpha} u\right\|_{M, Q}
$$

Thus both sides of the last inequality are equivalent norms on $W_{0}^{1, x} L_{M}(Q)$. 
We then have the following complementary system:

$$
\left(\begin{array}{ll}
W_{0}^{1, x} L_{M}(Q) & F \\
W_{0}^{1, x} E_{M}(Q) & F_{0}
\end{array}\right),
$$

$F$ being the dual space of $W_{0}^{1, x} E_{M}(Q)$ and $W_{0}^{1, x} L_{M}(Q)$ being the dual space of $F_{0} . F$ is also, up to isomorphism, the quotient of $\prod L_{\bar{M}}$ by the polar set $W_{0}^{1, x} E_{M}(Q)^{\perp}$, and it will be denoted by $F=W^{-1, x} L_{\bar{M}}(Q)$; moreover, it is known that

$$
W^{-1, x} L_{\bar{M}}(Q)=\left\{f=\sum_{|\alpha| \leq 1} D_{x}^{\alpha} f_{\alpha}: f_{\alpha} \in L_{\bar{M}}(Q)\right\} .
$$

This space will be equipped with the usual quotient norm:

$$
\|f\|=\inf \sum_{|\alpha| \leq 1}\left\|f_{\alpha}\right\|_{\bar{M}, Q}
$$

where the inf is taken over all possible decompositions $f=\sum_{|\alpha| \leq 1} D_{x}^{\alpha} f_{\alpha}$, $f_{\alpha} \in L_{\bar{M}}(Q)$. The space $F_{0}$ is then given by $F_{0}=\left\{f=\sum_{|\alpha| \leq 1} D_{x}^{\alpha} f_{\alpha}: f_{\alpha} \in\right.$ $\left.E_{\bar{M}}(Q)\right\}$ and is denoted by $F_{0}=W^{-1, x} E_{\bar{M}}(Q)$.

2.7. We will use the following technical lemmas:

Lemma 1 (see Gossez [10]). Let $\Omega$ be a bounded open subset of $\mathbb{R}^{N}$ and let $\left(\varrho_{\sigma}\right)$ be a mollifier sequence in $\mathbb{R}^{N}$. Denote by $u_{\sigma}=\widetilde{u} * \varrho_{\sigma}$ the mollification of $u$, where $\widetilde{u}$ is the zero extension of $u$. If $u \in L_{M}(\Omega)$ then $u_{\sigma} \in L_{M}(\Omega)$, and if $2 u \in \mathcal{L}(\Omega)$, we have

$$
\int_{\Omega} M\left(u_{\sigma}-u\right) d x \rightarrow 0 \quad \text { as } \sigma \rightarrow 0 .
$$

Lemma 2 (see Morrey [17]). If $u \in W_{0}^{1,1}(\Omega)$ then $\left\|u_{\sigma}-u\right\|_{1, \Omega} \leq \sigma\|\nabla u\|_{1, \Omega}$.

Lemma 3. Let $M$ be an $N$-function and let $\left(u_{n}\right)$ be a bounded sequence in $W_{0}^{1, x} L_{M}(Q) \cap L^{\infty}\left(0, T ; L^{1}(\Omega)\right)$. If $u_{n}(t) \rightarrow u(t)$ weakly in $L^{1}(\Omega)$ for a.e. $t \in[0, T]$ then $u_{n} \rightarrow u$ strongly in $L^{1}(Q)$.

Proof. For each $v \in W_{0}^{1, x} L_{M}(Q)$ define $v_{\sigma}(x, t)=\int_{\mathbb{R}^{N}} v(y, t) \varrho_{\sigma}(x-y) d y$, where $v(y, t)=0$ if $y \notin \Omega$ and where $\left(\varrho_{\sigma}\right)$ is a mollifier sequence in $\mathbb{R}^{N}$.

Since $u_{n}(t) \rightarrow u(t)$ weakly in $L^{1}(\Omega)$ for a.e. $t \in[0, T]$ we have $u_{n \sigma} \rightarrow u_{\sigma}$ a.e. in $Q$ and $u_{n \sigma}(t) \rightarrow u_{\sigma}(t)$ strongly in $L^{1}(\Omega)$ for a.e. $t \in[0, T]$.

For all $n$ and $k$ and for a.e. $t \in[0, T]$ we have

$$
\begin{aligned}
\int_{\Omega}\left|u_{n}(t)-u_{k}(t)\right| d x \leq & \int_{\Omega}\left|u_{n}(t)-u_{n \sigma}(t)\right| d x+\int_{\Omega}\left|u_{n \sigma}(t)-u_{k \sigma}(t)\right| d x \\
& +\int_{\Omega}\left|u_{k \sigma}(t)-u_{k}(t)\right| d x \\
\leq & \sigma\left(\int_{\Omega}\left|\nabla u_{n}(t)\right| d x+\int_{\Omega}\left|\nabla u_{k}(t)\right| d x\right)+\left\|u_{n \sigma}(t)-u_{k \sigma}(t)\right\|_{1, \Omega} .
\end{aligned}
$$


Integrating this inequality over $[0, T]$ yields

$$
\begin{aligned}
\int_{Q}\left|u_{n}(t)-u_{k}(t)\right| d x d t \leq & \sigma\left(\int_{Q}\left|\nabla u_{n}(t)\right| d x d t+\int_{Q}\left|\nabla u_{k}(t)\right| d x d t\right) \\
& +\int_{0}^{T}\left\|u_{n \sigma}(t)-u_{k \sigma}(t)\right\|_{1, \Omega} d t
\end{aligned}
$$

which, by the continuous embedding $L_{M}(Q) \subset L^{1}(Q)$, gives

$$
\begin{aligned}
\int_{Q}\left|u_{n}(t)-u_{k}(t)\right| d x d t \leq & \sigma C_{1}\left(\left\|\nabla u_{n}\right\|_{M, Q}+\left\|\nabla u_{k}\right\|_{M, Q}\right) \\
& +\int_{0}^{T}\left\|u_{n \sigma}(t)-u_{k \sigma}(t)\right\|_{1, \Omega} d t,
\end{aligned}
$$

where $C_{1}$ and $C_{2}$ are constants, which do not depend on $n$ and $k$, such that

$$
\|\nabla v\|_{1, Q} \leq C_{1}\|\nabla v\|_{M, Q} \quad \forall v \in L_{M}(Q) \quad \text { and } \quad\left\|\nabla u_{n}\right\|_{M, Q} \leq C_{2} \quad \forall n .
$$

Consequently, we obtain

$$
\int_{Q}\left|u_{n}(t)-u_{k}(t)\right| d x \leq 2 C_{1} C_{2} \sigma+\int_{0}^{T}\left\|u_{n \sigma}(t)-u_{k \sigma}(t)\right\|_{1, \Omega} d t .
$$

Since $\left\|u_{n \sigma}(t)-u_{k \sigma}(t)\right\|_{1, \Omega} \rightarrow 0$ a.e. in $[0, T]$ as $n, k \rightarrow \infty$ and $\left\|u_{n \sigma}(t)\right\|_{L^{1}(\Omega)}$ $\leq\left\|u_{n}(t)\right\|_{L^{1}(\Omega)} \leq C$ uniformly with respect to $n$ and $t \in[0, T]$ we deduce by using Lebesgue's theorem that

$$
\int_{0}^{T}\left\|u_{n \sigma}(t)-u_{k \sigma}(t)\right\|_{1, \Omega} d t \rightarrow 0 \quad \text { as } n, k \rightarrow \infty,
$$

implying by the arbitrariness of $\sigma$ that $\int_{Q}\left|u_{n}(t)-u_{k}(t)\right| d x d t \rightarrow 0$ as $n, k \rightarrow \infty$. Hence $\left(u_{n}\right)$ is a Cauchy sequence in $L^{1}(Q)$ and thus $u_{n} \rightarrow u$ strongly in $L^{1}(Q)$.

3. Time mollification. For $u \in L_{M}(Q)$, define for all $\mu>0$ and all $(x, t) \in Q$,

$$
u_{\mu}(x, t)=\mu \int_{-\infty}^{t} \widetilde{u}(x, s) \exp (\mu(s-t)) d s,
$$

where $\widetilde{u}$ is the zero extension of $u$.

Proposition 1. If $u \in L_{M}(Q)$ then $u_{\mu}$ is measurable in $Q$ and $\partial u_{\mu} / \partial t$ $=\mu\left(u-u_{\mu}\right)$, and if $u \in \mathcal{L}_{M}(Q)$ then

$$
\int_{Q} M\left(u_{\mu}\right) d x d t \leq \int_{Q} M(u) d x d t
$$


Proof. Since $(x, t, s) \mapsto u(x, s) \exp (\mu(s-t))$ is measurable in $\Omega \times[0, T] \times$ $[0, T]$, by Fubini's theorem we deduce that $u_{\mu}$ is measurable.

By Jensen's integral inequality and the equality $\int_{-\infty}^{0} \mu \exp (\mu s) d s=1$, we have

$$
\begin{aligned}
M\left(\int_{-\infty}^{t} \mu \widetilde{u}(x, s) \exp (\mu(s-t)) d s\right) & =M\left(\int_{-\infty}^{0} \mu \exp (\mu s) \widetilde{u}(x, s+t) d s\right) \\
& \leq \int_{-\infty}^{0} \mu \exp (\mu s) M(\widetilde{u}(x, s+t)) d s
\end{aligned}
$$

which implies

$$
\begin{aligned}
\int_{Q} M\left(u_{\mu}(x, t)\right) d x d t & \leq \int_{\Omega \times \mathbb{R}}\left(\int_{-\infty}^{0} \mu \exp (\mu s) M(\widetilde{u}(x, s+t)) d s\right) d x d t \\
& \leq \int_{-\infty}^{0} \mu \exp (\mu s)\left(\int_{\Omega \times \mathbb{R}} M(\widetilde{u}(x, s+t)) d x d t\right) d s \\
& \leq \int_{-\infty}^{0} \mu \exp (\mu s)\left(\int_{Q} M(u(x, t)) d x d t\right) d s \leq \int_{Q} M(u) d x d t
\end{aligned}
$$

Furthermore, for a.e. $(x, t) \in Q$,

$$
\begin{aligned}
\frac{\partial u_{\mu}}{\partial t}(x, t) & =\lim _{\theta \rightarrow 0} \frac{1}{\theta}\left(e^{-\mu \theta}-1\right) u_{\mu}(x, t)+\lim _{\theta \rightarrow 0} \frac{1}{\theta} \int_{t}^{t+\theta} u(x, s) e^{\mu(s-(t+\theta))} d s \\
& =-\mu u_{\mu}(x, t)+\mu u(x, t) . \text { - }
\end{aligned}
$$

Proposition 2. (i) If $u \in L_{M}(Q)$ then $u_{\mu} \rightarrow u$ as $\mu \rightarrow \infty$ in $L_{M}(Q)$ for the modular convergence.

(ii) If $u \in W^{1, x} L_{M}(Q)$ then $u_{\mu} \rightarrow u$ as $\mu \rightarrow \infty$ in $W^{1, x} L_{M}(Q)$ for the modular convergence.

Proof. (i) Let $\left(\varphi_{k}\right) \subset \mathcal{D}(Q)$ be such that $\varphi_{k} \rightarrow u$ in $L_{M}(Q)$ for the modular convergence. Let $\lambda>0$ be large enough such that

$$
\frac{u}{\lambda} \in \mathcal{L}_{M}(Q) \text { and } \int_{Q} M\left(\frac{\varphi_{k}-u}{\lambda}\right) d x d t \rightarrow 0 \quad \text { as } k \rightarrow \infty .
$$

For a.e. $(x, t) \in Q$ we have

$$
\left|\left(\varphi_{k}\right)_{\mu}(x, t)-\varphi_{k}(x, t)\right|=\frac{1}{\mu}\left|\frac{\partial \varphi_{k}}{\partial t}(x, t)\right| \leq \frac{1}{\mu}\left\|\frac{\partial \varphi_{k}}{\partial t}\right\|_{\infty} .
$$

On the other hand, 


$$
\begin{aligned}
& \int_{Q} M\left(\frac{u_{\mu}-u}{3 \lambda}\right) d x d t \\
& \leq \frac{1}{3} \int_{Q} M\left(\frac{u_{\mu}-\left(\varphi_{k}\right)_{\mu}}{\lambda}\right) d x d t+\frac{1}{3} \int_{Q} M\left(\frac{\left(\varphi_{k}\right)_{\mu}-\varphi_{k}}{\lambda}\right) d x d t \\
&+\frac{1}{3} \int_{Q} M\left(\frac{\varphi_{k}-u}{\lambda}\right) d x d t \\
& \leq \frac{1}{3} \int_{Q} M\left(\frac{\left(\varphi_{k}-u\right)_{\mu}}{\lambda}\right) d x d t+\frac{1}{3} \int_{Q} M\left(\frac{\left(\varphi_{k}\right)_{\mu}-\varphi_{k}}{\lambda}\right) d x d t \\
&+\frac{1}{3} \int_{Q} M\left(\frac{\varphi_{k}-u}{\lambda}\right) d x d t
\end{aligned}
$$

This implies that

$$
\int_{Q} M\left(\frac{u_{\mu}-u}{3 \lambda}\right) d x d t \leq \frac{2}{3} \int_{Q} M\left(\frac{\varphi_{k}-u}{\lambda}\right) d x d t+\frac{1}{3} M\left(\frac{1}{\mu \lambda}\left\|\frac{\partial \varphi_{k}}{\partial t}\right\|_{\infty}\right) \operatorname{meas}(Q) .
$$

Let $\varepsilon>0$. There exist $k$ and $\mu_{0}$ such that

$$
\int_{Q} M\left(\frac{\varphi_{k}-u}{\lambda}\right) d x d t \leq \varepsilon
$$

and

$$
M\left(\frac{1}{\mu \lambda}\left\|\frac{\partial \varphi_{k}}{\partial t}\right\|_{\infty}\right) \operatorname{meas}(Q) \leq \varepsilon \quad \text { for all } \mu \geq \mu_{0}
$$

Hence

$$
\int_{Q} M\left(\frac{u_{\mu}-u}{3 \lambda}\right) d x d t \leq \varepsilon \quad \text { for all } \mu \geq \mu_{0} .
$$

(ii) Since for any $\alpha$ with $|\alpha| \leq 1$, we have $D_{x}^{\alpha}\left(u_{\mu}\right)=\left(D_{x}^{\alpha} u\right)_{\mu}$, the first part above applied to each $D_{x}^{\alpha} u$ gives the result.

REMARK 1. If $u \in E_{M}(Q)$, we can choose $\lambda$ arbitrarily small since $\mathcal{D}(Q)$ is (norm) dense in $E_{M}(Q)$. Thus, for all $\lambda>0$,

$$
\int_{Q} M\left(\frac{u_{\mu}-u}{\lambda}\right) d x d t \rightarrow 0 \quad \text { as } \mu \rightarrow \infty
$$

and $u_{\mu} \rightarrow u$ strongly in $E_{M}(Q)$. The same remark is true if one replaces $E_{M}(Q)$ with $W^{1, x} E_{M}(Q)$.

Proposition 3. If $u_{n} \rightarrow u$ in $W^{1, x} L_{M}(Q)$ strongly (resp. for the modular convergence) then $\left(u_{n}\right)_{\mu} \rightarrow u_{\mu}$ in $W^{1, x} L_{M}(Q)$ strongly (resp. for the modular convergence). 
Proof. It suffices to prove the proposition for the zero order derivative. For all (resp. some) $\lambda>0$,

$$
\int_{Q} M\left(\frac{\left(u_{n}\right)_{\mu}-u_{\mu}}{\lambda}\right) d x d t \leq \int_{Q} M\left(\frac{u_{n}-u}{\lambda}\right) d x d t \rightarrow 0 \quad \text { as } n \rightarrow \infty
$$

so $\left(u_{n}\right)_{\mu} \rightarrow u_{\mu}$ in $L_{M}(Q)$ strongly (resp. for the modular convergence).

4. Existence result. Let $\Omega$ be a bounded open subset of $\mathbb{R}^{N}$ with the segment property, $T>0$, and set $Q=\Omega \times(0, T)$. Let $M$ and $P$ be two N-functions such that $P \ll M$.

Consider a second order operator $A: D(A) \subset W^{1, x} L_{M}(Q) \rightarrow W^{-1, x} L_{\bar{M}}(Q)$ of the form

$$
A(u)=-\operatorname{div}(a(x, t, u, \nabla u))+a_{0}(x, t, u, \nabla u)
$$

where $a: \Omega \times[0, T] \times \mathbb{R} \times \mathbb{R}^{N} \rightarrow \mathbb{R}^{N}$ and $a_{0}: \Omega \times[0, T] \times \mathbb{R} \times \mathbb{R}^{N} \rightarrow \mathbb{R}$ are Carathéodory functions satisfying for a.e. $(x, t) \in \Omega \times[0, T]$ and all $s \in \mathbb{R}$, $\xi \neq \xi^{*} \in \mathbb{R}^{N}$ :

$$
\begin{gathered}
|a(x, t, s, \xi)| \leq \beta\left(c(x, t)+\bar{M}^{-1} P(\gamma(|s|))+\bar{M}^{-1} M(\gamma|\xi|)\right), \\
\left|a_{0}(x, t, s, \xi)\right| \leq \beta\left(c(x, t)+\bar{M}^{-1} P(\gamma(|s|))+\bar{M}^{-1} P(\gamma|\xi|)\right), \\
{\left[a(x, t, s, \xi)-a\left(x, t, s, \xi^{*}\right)\right]\left[\xi-\xi^{*}\right]>0} \\
a(x, t, s, \xi) \xi+a_{0}(x, t, s, \xi) s \geq \alpha M(|\xi| / \lambda)-d(x, t),
\end{gathered}
$$

where $c(x, t) \in E_{\bar{M}}(Q), c \geq 0 ; d(x, t) \in L^{1}(Q) ; \alpha, \beta, \gamma>0$.

Furthermore let

$$
f \in W^{-1, x} E_{\bar{M}}(Q)
$$

Consider the following parabolic initial-boundary value problem:

$$
\begin{cases}\partial u / \partial t+A(u)=f & \text { in } Q, \\ u(x, t)=0 & \text { on } \partial \Omega \times(0, T), \\ u(x, 0)=u_{0}(x) & \text { in } \Omega,\end{cases}
$$

where $u_{0}$ is a given function in $L^{2}(\Omega)$.

We shall prove the following existence theorem:

THEOREM 1. Assume that (4)-(8) hold true. Then there exists at least one weak solution $u \in W_{0}^{1, x} L_{M}(Q) \cap C\left([0, T], L^{2}(\Omega)\right)$ of $(9)$ in the following sense:

$$
\begin{aligned}
-\int_{Q} u \frac{\partial \varphi}{\partial t} d x d t+\left[\int_{\Omega} u(t) \varphi(t) d x\right]_{0}^{T} & +\int_{Q} a(x, t, u, \nabla u) \cdot \nabla \varphi d x d t \\
& +\int_{Q} a_{0}(x, t, u, \nabla u) \varphi d x d t=\langle f, \varphi\rangle
\end{aligned}
$$

for all $\varphi \in C^{1}\left([0, T], L^{2}(\Omega)\right)$. 
REMARK 2. As in the elliptic case (see [9] and [11]), the introduction of $P$ instead of $M$ in (4) and (5) is done only to guarantee the boundedness in $L_{\bar{M}}(Q)$ of $\bar{M}^{-1} P\left(\gamma\left|u_{n}\right|\right)$ and $\bar{M}^{-1} P\left(\gamma\left|\nabla u_{n}\right|\right)$ whenever $u_{n}$ is bounded in $W^{1, x} L_{M}(Q)$. In the elliptic case, one usually takes $P=M$ in the term $\bar{M}^{-1} P\left(\gamma\left|u_{n}\right|\right)$ since $u_{n}$ is bounded in a smaller space $L_{R}(\Omega)$ with $M \ll R$ (see $[9]$ ).

In the parabolic case, we cannot however deduce the same boundedness. Nevertheless, we can take $P=M$ if one of the following assertions holds:

1) $M$ satisfies the $\Delta_{2}$ condition near infinity.

2) $A$ is monotone, i.e. $\langle A(u)-A(v), u-v\rangle \geq 0$ for all $u, v \in D(A) \cap$ $W_{0}^{1, x} L_{M}(Q)$.

3) $M$ grows essentially less rapidly than the $\mathrm{N}$-function $\bar{M} \circ M$.

Indeed, suppose first that $M$ satisfies the $\Delta_{2}$ condition. Then (4) and (5), with now $P=M$, imply that for all $\varepsilon>0$,

$$
\begin{aligned}
|a(x, t, s, \xi)| & \leq \beta_{\varepsilon}\left(c_{\varepsilon}(x, t)+\bar{M}^{-1} M(\varepsilon|s|)+\bar{M}^{-1} M(\varepsilon|\xi|)\right), \\
\left|a_{0}(x, t, s, \xi)\right| & \leq \beta_{\varepsilon}\left(c_{\varepsilon}(x, t)+\bar{M}^{-1} M(\varepsilon|s|)+\bar{M}^{-1} M(\varepsilon|\xi|)\right),
\end{aligned}
$$

which allows us to deduce the boundedness in $L_{\bar{M}}(Q)$ of $a\left(x, t, u_{n}, \nabla u_{n}\right)$ and $a_{0}\left(x, t, u_{n}, \nabla u_{n}\right)$.

In the case where $A$ is monotone, for all $\varphi \in W_{0}^{1, x} E_{M}(Q)$ we have

$$
\left\langle A\left(u_{n}\right)-A(\varphi), u_{n}-\varphi\right\rangle \geq 0
$$

where $\langle$,$\rangle is the pairing between W_{0}^{1, x} L_{M}(Q)$ and $W^{-1, x} L_{\bar{M}}(Q)$. This gives

$$
\left\langle A\left(u_{n}\right), \varphi\right\rangle \leq\left\langle A\left(u_{n}\right), u_{n}\right\rangle-\left\langle A(\varphi), u_{n}-\varphi\right\rangle,
$$

implying that, since $u_{n}$ is bounded in $W_{0}^{1, x} L_{M}(Q)$ and $\left\langle A\left(u_{n}\right), u_{n}\right\rangle \leq C_{1}$ thanks to the a priori estimates,

$$
\left\langle A\left(u_{n}\right), \varphi\right\rangle \leq C_{\varphi} \quad \text { for all } \varphi \in W_{0}^{1, x} E_{M}(Q) .
$$

Therefore, the Banach-Steinhaus theorem yields the boundedness of $A\left(u_{n}\right)$ in $W^{-1, x} L_{\bar{M}}(Q)$.

Assume, finally, that $M \ll \bar{M} \circ M$. Then for all $\varepsilon>0$ there is $t_{\varepsilon} \geq 0$ such that

$$
M(\gamma t) \leq \bar{M}\left(M\left(\varepsilon^{2} t\right)\right) \quad \text { for all } t \geq t_{\varepsilon}
$$

implies that

$$
\begin{aligned}
|a(x, t, s, \xi)| & \leq \beta\left(c_{\varepsilon}(x, t)+\varepsilon M(\varepsilon|s|)+\varepsilon M(\varepsilon|\xi|)\right), \\
\left|a_{0}(x, t, s, \xi)\right| & \leq \beta\left(c_{\varepsilon}(x, t)+\varepsilon M(\varepsilon|s|)+\varepsilon M(\varepsilon|\xi|)\right),
\end{aligned}
$$

which gives the boundedness and the weak convergence in $L^{1}(Q)$ of $a\left(x, t, u_{n}, \nabla u_{n}\right)$ and $a_{0}\left(x, t, u_{n}, \nabla u_{n}\right)$. This leads to

$$
u_{n}(t) \rightarrow u(t) \quad \text { a.e. in } \Omega \text { and then } u_{n} \rightarrow u \text { in } L^{1}(Q) .
$$


Hence, the proof below can be adapted to this situation by proving the existence of an entropy solution of (9).

Note that there are $N$-functions $M$ for which $M$ and $\bar{M} \circ M$ are equivalent. Indeed, take $M(t)=\exp (t)$ near infinity. We have $\bar{M}(t)=t \log t$ near infinity and so $(\bar{M} \circ M)(t)=t \exp (t)$ is equivalent to $M(t)$ since $M(t) \leq(\bar{M} \circ M)(t) \leq M(2 t)$ for $t$ large enough.

Proof of Theorem 1. For convenience we suppose that $u_{0}=0$. The general case can be handled similarly.

We will use a Galerkin method due to Landes and Mustonen [15]. For the Galerkin method we choose the sequence $\left\{w_{1}, w_{2}, \ldots\right\}$ in $\mathcal{D}(\Omega)$ such that $\bigcup_{n=1}^{\infty} V_{n}$ with

$$
V_{n}=\operatorname{span}\left\{w_{1}, \ldots, w_{n}\right\}
$$

is dense in $W_{0}^{j} L_{M}(\Omega)$ for the modular convergence, where $j>q(M, N)$ is taken such that $W_{0}^{j} L_{M}(\Omega)$ is continuously embedded in $C^{1}(\bar{\Omega})$.

For any $v \in W_{0}^{j} L_{M}(\Omega)$ there exists a sequence $\left(v_{k}\right) \subset \bigcup_{n=1}^{\infty} V_{n}$ such that $v_{k} \rightarrow v$ in $W_{0}^{j} L_{M}(\Omega)$ for the modular convergence.

We set further $\mathcal{V}_{n}=C\left([0, T], V_{n}\right)$. It is easy to see that the closure of $\bigcup_{n=1}^{\infty} \mathcal{V}_{n}$ with respect to the norm

$$
\|v\|_{C^{1,0}(Q)}=\sup _{|\alpha| \leq 1}\left\{\left|D^{\alpha} v(x, t)\right|:(x, t) \in Q\right\}
$$

contains $\mathcal{D}(Q)$. This implies that for any $f \in W^{-1, x} E_{\bar{M}}(Q)$ there exists a sequence $\left(f_{k}\right) \subset \bigcup_{n=1}^{\infty} \mathcal{V}_{n}$ such that $f_{k} \rightarrow f$ strongly in $W^{-1, x} E_{\bar{M}}(Q)$.

For any $u_{0} \in L^{2}(\Omega)$ there is a sequence $\left(u_{0 k}\right) \subset \bigcup_{n=1}^{\infty} V_{n}$ such that $u_{0 k} \rightarrow u_{0}$ in $L^{2}(\Omega)$.

We divide the proof into three steps.

STEP 1. A priori estimates. As in [15], by using Lemma 1 of [14], there exists a Galerkin solution $u_{n}$ of $(9)$ in the following sense:

$$
\left\{\begin{array}{l}
u_{n} \in \mathcal{V}_{n}, \frac{\partial u_{n}}{\partial t} \in L^{1}\left(0, T ; V_{n}\right), u_{n}(0)=u_{0 n}, \text { and for all } \varphi \in \mathcal{V}_{n} \\
\int_{Q_{\tau}} \frac{\partial u_{n}}{\partial t} \varphi d x d t+\int_{Q_{\tau}} a\left(x, t, u_{n}, \nabla u_{n}\right) \nabla \varphi d x d t \\
\quad+\int_{Q_{\tau}} a_{0}\left(x, t, u_{n}, \nabla u_{n}\right) \varphi d x d t=\int_{Q_{\tau}} f_{n} \varphi d x d t
\end{array}\right.
$$

for all $\tau \in(0, T)$, where $Q_{\tau}=\Omega \times(0, \tau)$.

Letting $\varphi=u_{n}$ in (10) and using (4) and (7) yields

$$
\begin{gathered}
\left\|u_{n}\right\|_{W_{0}^{1, x} L_{M}(Q)} \leq C, \quad\left\|u_{n}\right\|_{L^{\infty}\left(0, T ; L^{2}(\Omega)\right)} \leq C, \\
\int_{Q}\left[a\left(x, t, u_{n}, \nabla u_{n}\right) \nabla u_{n}+a_{0}\left(x, t, u_{n}, \nabla u_{n}\right) u_{n}\right] d x d t \leq C ;
\end{gathered}
$$

here and below, $C$ is a constant not depending on $n$. 
Using (5) and the fact that $P \ll M$, it is easy to see that $a_{0}\left(x, t, u_{n}, \nabla u_{n}\right)$ is bounded in $L_{\bar{M}}(Q)$. This implies that

$$
\int_{Q} a\left(x, t, u_{n}, \nabla u_{n}\right) \nabla u_{n} d x d t \leq C .
$$

To prove that $a\left(x, t, u_{n}, \nabla u_{n}\right)$ is bounded in $\left(L_{\bar{M}}(Q)\right)^{N}$, let $\varphi \in\left(E_{M}(Q)\right)^{N}$ and $\|\varphi\|_{M, Q}=1$. By (6), we have

$$
\int_{Q}\left[a\left(x, t, u_{n}, \nabla u_{n}\right)-a\left(x, t, u_{n}, \varphi\right)\right]\left[\nabla u_{n}-\varphi\right] d x d t \geq 0,
$$

which gives

$$
\begin{aligned}
\int_{Q} a\left(x, t, u_{n}, \nabla u_{n}\right) \varphi d x d t \leq & \int_{Q} a\left(x, t, u_{n}, \nabla u_{n}\right) \nabla u_{n} d x d t \\
& -\int_{Q} a\left(x, t, u_{n}, \varphi\right)\left[\nabla u_{n}-\varphi\right] d x d t .
\end{aligned}
$$

Since $a\left(x, t, u_{n}, \varphi\right)$ is uniformly bounded in $\left(L_{\bar{M}}(Q)\right)^{N}$, thanks to (4), we deduce that

$$
\int_{Q} a\left(x, t, u_{n}, \nabla u_{n}\right) \varphi d x d t \leq C \quad \text { for all } \varphi \in\left(E_{M}(Q)\right)^{N},\|\varphi\|_{M, Q}=1 .
$$

Using the dual norm of $\left(L_{\bar{M}}(Q)\right)^{N}$ we conclude that $a\left(x, t, u_{n}, \nabla u_{n}\right)$ is bounded in $\left(L_{\bar{M}}(Q)\right)^{N}$.

Hence, for a subsequence,

$\left\{\begin{array}{l}u_{n} \rightarrow u \text { weakly in } W_{0}^{1, x} L_{M}(Q) \text { for } \sigma\left(\prod L_{M}, \prod E_{\bar{M}}\right) \text { and weakly in } L^{2}(Q), \\ a_{0}\left(x, t, u_{n}, \nabla u_{n}\right) \rightarrow h_{0}, a\left(x, t, u_{n}, \nabla u_{n}\right) \rightarrow h \text { in } L_{\bar{M}}(Q) \text { for } \sigma\left(\prod L_{\bar{M}}, \prod E_{M}\right)\end{array}\right.$ for some $h_{0} \in L_{\bar{M}}(Q)$ and some $h \in\left(L_{\bar{M}}(Q)\right)^{N}$.

As in [15], by using Lemma 3 we deduce that $u_{n} \rightarrow u$ strongly in $L^{1}(Q)$ and for some subsequence $u_{n}(x, t) \rightarrow u(x, t)$ a.e. in $Q$.

STEP 2. Almost everywhere convergence of the gradients. For all $\varphi \in$ $C^{1}([0, T], \mathcal{D}(\Omega))$, from (10) we get

$$
\begin{aligned}
-\int_{Q} u \frac{\partial \varphi}{\partial t} d x d t+\left[\int_{\Omega} u(t) \varphi(t) d x\right]_{0}^{T} & \\
& +\int_{Q} h \cdot \nabla \varphi d x d t+\int_{Q} h_{0} \varphi d x d t=\langle f, \varphi\rangle .
\end{aligned}
$$

Let $\left(\phi_{j}\right) \subset \mathcal{D}(Q)$ be such that $\phi_{j} \rightarrow u$ in $L^{2}(Q)$ and in $W_{0}^{1, x} L_{M}(Q)$ for the modular convergence. For $\mu \in \mathbb{N}$, let

$$
\left(T_{l}\left(\phi_{j}\right)\right)_{\mu}(x, t)=\mu \int_{-\infty}^{t} T_{l}\left(\phi_{j}\right)(x, s) \exp (\mu(s-t)) d s
$$


where $T_{l}$ is the usual truncation at height $l$ defined by

$$
T_{l}(s)= \begin{cases}s & \text { if }|s| \leq l \\ T_{l}(s)=l s /|s| & \text { if }|s|>l\end{cases}
$$

Then $\left(T_{l}\left(\phi_{j}\right)\right)_{\mu} \rightarrow T_{l}\left(\phi_{j}\right)$ in $W_{0}^{1, x} L_{M}(Q)$ strongly as $\mu \rightarrow \infty$ and

$$
\frac{\partial}{\partial t}\left(T_{l}\left(\phi_{j}\right)\right)_{\mu}=\mu\left(T_{l}\left(\phi_{j}\right)-\left(T_{l}\left(\phi_{j}\right)\right)_{\mu}\right) \text {. }
$$

Take the mollification with respect to the space variable, $\left[\left(T_{l}\left(\phi_{j}\right)\right)_{\mu}\right]_{\sigma}$ for $\sigma>0$. It is obvious that this sequence is in $C^{1}([0, T], \mathcal{D}(\Omega))$. Finally, choose $v_{k}$ as a diagonal sequence of $\left[\left(T_{l}\left(\phi_{j}\right)\right)_{\mu}\right]_{\sigma}$ such that $v_{k} \rightarrow u$ in $W_{0}^{1, x} L_{M}(Q)$ for the modular convergence.

Indeed, let $\lambda>0$ be such that

$$
\frac{1}{\lambda} D_{x}^{\alpha} u \in \mathcal{L}_{M}(Q), \quad \int_{Q} M\left(\frac{D_{x}^{\alpha} \phi_{j}-D_{x}^{\alpha} u}{\lambda}\right) d x d t \rightarrow 0, \forall|\alpha| \leq 1 .
$$

We have

$$
\begin{aligned}
\int_{Q} M\left(\frac{D_{x}^{\alpha} v_{k}-D_{x}^{\alpha} u}{4 \lambda}\right) d x d t \leq & \int_{Q} M\left(\frac{D_{x}^{\alpha}\left[\left(T_{l}\left(\phi_{j}\right)\right)_{\mu}\right]_{\sigma}-D_{x}^{\alpha}\left(T_{l}\left(\phi_{j}\right)\right)_{\mu}}{\lambda}\right) d x d t \\
& +\int_{Q} M\left(\frac{D_{x}^{\alpha}\left(T_{l}\left(\phi_{j}\right)\right)_{\mu}-D_{x}^{\alpha} T_{l}\left(\phi_{j}\right)}{\lambda}\right) d x d t \\
& +\int_{Q} M\left(\frac{D_{x}^{\alpha} T_{l}\left(\phi_{j}\right)-D_{x}^{\alpha} \phi_{j}}{\lambda}\right) d x d t \\
& +\int_{Q} M\left(\frac{D_{x}^{\alpha} \phi_{j}-D_{x}^{\alpha} u}{\lambda}\right) d x d t .
\end{aligned}
$$

The first three integrals of the right side go to 0 since $D_{x}^{\alpha}\left[\left(T_{l}\left(\phi_{j}\right)\right)_{\mu}\right]_{\sigma}$, $D_{x}^{\alpha}\left(T_{l}\left(\phi_{j}\right)\right)_{\mu}$ and $D_{x}^{\alpha} T_{l}\left(\phi_{j}\right)$ are strongly convergent in $W_{0}^{1, x} E_{M}(Q)$ respectively as $\sigma \rightarrow 0, \mu \rightarrow \infty$ and $l \rightarrow \infty$ by using the facts that $\left(T_{l}\left(\phi_{j}\right)\right)_{\mu}, T_{l}\left(\phi_{j}\right)$ and $\phi_{j}$ are in $W_{0}^{1, x} E_{M}(Q)$ (see Lemma 5 of [10]).

Since the last integral goes to 0 by (12), we deduce that $v_{k} \rightarrow u$ in $W_{0}^{1, x} L_{M}(Q)$ for the modular convergence and hence, for a subsequence, $v_{k} \rightarrow u, \nabla v_{k} \rightarrow \nabla u$ a.e. in $Q$ and weakly in $L_{M}(Q)$ for $\sigma\left(\prod L_{M}, \prod L_{\bar{M}}\right)$.

On the other hand, setting as in [15], $Q_{l}=\{(x, t) \in Q:|u(x, t)| \leq l\}$, we have

$$
T_{l}(u)=u \text { in } Q_{l}, \quad \operatorname{sgn}\left(T_{l}(u)-\left(T_{l}(u)\right)_{\mu}\right)=\operatorname{sgn}\left(u-\left(T_{l}(u)\right)_{\mu}\right) \text { in } Q \backslash Q_{l} .
$$


Therefore, as in [15],

$$
\begin{aligned}
\int_{Q} \frac{\partial v_{k}}{\partial t}\left(v_{k}-u\right) d x d t & =\mu \int_{Q}\left\{\left(T_{l}\left(\phi_{j}\right)\right)_{\sigma}-\left[\left(T_{l}\left(\phi_{j}\right)\right)_{\sigma}\right]_{\mu}\right\}\left\{\left[\left(T_{l}\left(\phi_{j}\right)\right)_{\sigma}\right]_{\mu}-u\right\} d x d t \\
& \rightarrow \mu \int_{Q}\left\{T_{l}(u)-\left(T_{l}(u)\right)_{\mu}\right\}\left\{\left(T_{l}(u)\right)_{\mu}-u\right\} d x d t \\
& =-\mu \int_{Q_{l}}\left(u-\left(T_{l}(u)\right)_{\mu}\right)^{2} d x d t \\
& +\mu \int_{Q \backslash Q_{l}}\left\{T_{l}(u)-\left(T_{l}(u)\right)_{\mu}\right\}\left\{\left(T_{l}(u)\right)_{\mu}-u\right\} d x d t \leq 0
\end{aligned}
$$

as $\sigma \rightarrow 0$ and $j \rightarrow \infty$, for any $\mu$ and $l$. Consequently,

$$
\limsup _{k \rightarrow \infty} \int_{Q} \frac{\partial v_{k}}{\partial t}\left(v_{k}-u_{n}\right) d x d t \leq 0
$$

and then

$$
\limsup _{k \rightarrow \infty} \limsup _{n \rightarrow \infty} \int_{Q} \frac{\partial v_{k}}{\partial t}\left(v_{k}-u_{n}\right) d x d t \leq 0, \quad \text { since } \quad \frac{\partial v_{k}}{\partial t} \in E_{\bar{M}}(Q) .
$$

This implies that

$$
\limsup _{k \rightarrow \infty} \limsup _{n \rightarrow \infty} \int_{Q} \frac{\partial u_{n}}{\partial t}\left(v_{k}-u_{n}\right) d x d t \leq 0
$$

since

$$
\begin{aligned}
\int_{Q} \frac{\partial u_{n}}{\partial t}\left(v_{k}-u_{n}\right) d x d t & =-\frac{1}{2} \int_{Q} \frac{\partial}{\partial t}\left(u_{n}(t)-v_{k}(t)\right)^{2} d x d t+\int_{Q} \frac{\partial v_{k}}{\partial t}\left(v_{k}-u_{n}\right) d x d t \\
& =-\frac{1}{2}\left\|u_{n}(T)-v_{k}(T)\right\|_{L^{2}(\Omega)}^{2}+\int_{Q} \frac{\partial v_{k}}{\partial t}\left(v_{k}-u_{n}\right) d x d t
\end{aligned}
$$

From (10) and (11) we have

$$
\begin{aligned}
& \limsup _{n \rightarrow \infty}\left(\int_{Q}\left(a\left(x, t, u_{n}, \nabla u_{n}\right) \nabla u_{n}-h \nabla v_{k}+a_{0}\left(x, t, u_{n}, \nabla u_{n}\right) u_{n}-h_{0} v_{k}\right) d x d t\right) \\
& \leq \limsup _{n \rightarrow \infty}\left\langle f_{n}, u_{n}\right\rangle-\left\langle f, v_{k}\right\rangle+\limsup _{n \rightarrow \infty}\left(-\int_{Q} \frac{\partial u_{n}}{\partial t} u_{n} d x d t\right)-\int_{Q} \frac{\partial v_{k}}{\partial t} u d x d t \\
& \quad+\left[\int_{\Omega} u(t) v_{k}(t) d x\right]_{0}^{T} \\
& =\left\langle f, u-v_{k}\right\rangle+\limsup _{n \rightarrow \infty} \int_{Q} \frac{\partial u_{n}}{\partial t}\left(v_{k}-u_{n}\right) d x d t
\end{aligned}
$$


where we have used the fact that

$$
\begin{aligned}
-\int_{Q} \frac{\partial v_{k}}{\partial t} u d x d t+\left[\int_{\Omega} u(t) v_{k}(t) d x\right]_{0}^{T} \\
=\lim _{n \rightarrow \infty}\left(-\int_{Q} \frac{\partial v_{k}}{\partial t} u_{n} d x d t+\left[\int_{\Omega} u_{n}(t) v_{k}(t) d x\right]_{0}^{T}\right) \\
=\lim _{n \rightarrow \infty} \int_{Q} \frac{\partial u_{n}}{\partial t} v_{k} d x d t
\end{aligned}
$$

We deduce that

$$
\begin{array}{r}
\limsup _{k \rightarrow \infty} \limsup _{n \rightarrow \infty}\left(\int _ { Q } \left(a\left(x, t, u_{n}, \nabla u_{n}\right) \nabla u_{n}-h \nabla v_{k}\right.\right. \\
\left.\left.+a_{0}\left(x, t, u_{n}, \nabla u_{n}\right) u_{n}-h_{0} v_{k}\right) d x d t\right) \\
\leq \limsup _{k \rightarrow \infty}\left\langle f, u-v_{k}\right\rangle+\limsup _{k \rightarrow \infty} \limsup _{n \rightarrow \infty} \int_{Q} \frac{\partial u_{n}}{\partial t}\left(v_{k}-u_{n}\right) d x d t \leq 0
\end{array}
$$

which implies that

$$
\begin{aligned}
& \limsup _{k \rightarrow \infty} \limsup _{n \rightarrow \infty}\left(\int _ { Q } \left(a\left(x, t, u_{n}, \nabla u_{n}\right)\left[\nabla u_{n}-\nabla v_{k}\right]\right.\right. \\
& \left.\left.+a_{0}\left(x, t, u_{n}, \nabla u_{n}\right)\left(u_{n}-v_{k}\right)\right) d x d t\right) \leq 0
\end{aligned}
$$

since, as can be easily seen,

$$
\begin{aligned}
\lim _{n \rightarrow \infty} \int_{Q}\left(a\left(x, t, u_{n}, \nabla u_{n}\right) \nabla v_{k}+a_{0}\left(x, t, u_{n}, \nabla u_{n}\right)\right. & \left.v_{k}\right) d x d t \\
& =\int_{Q}\left(h \nabla v_{k}+h_{0} v_{k}\right) d x d t
\end{aligned}
$$

For any $r>0$ and $k \in \mathbb{N}$, we denote by $\chi_{k}^{r}$ and $\chi^{r}$ the characteristic functions of $\left\{(x, t) \in Q:\left|\nabla v_{k}\right| \leq r\right\}$ and $\{(x, t) \in Q:|\nabla u| \leq r\}$, respectively.

For any $l>0$, we have

$$
\begin{aligned}
& \int_{\left\{\left|u_{n}\right| \leq l\right\}}\left[a\left(x, t, u_{n}, \nabla u_{n}\right)-a\left(x, t, u_{n}, \nabla u \cdot \chi^{s}\right)\right]\left[\nabla u_{n}-\nabla u \cdot \chi^{s}\right] d x d t \\
& \quad-\int_{\left\{\left|u_{n}\right| \leq l\right\}}\left[a\left(x, t, u_{n}, \nabla u_{n}\right)-a\left(x, t, u_{n}, \nabla v_{k} \cdot \chi_{k}^{s}\right)\right]\left[\nabla u_{n}-\nabla v_{k} \cdot \chi_{k}^{s}\right] d x d t
\end{aligned}
$$




$$
\begin{aligned}
& =\int_{\left\{\left|u_{n}\right| \leq l\right\}} a\left(x, t, u_{n}, \nabla u \cdot \chi^{s}\right)\left[\nabla u_{n}-\nabla u \cdot \chi^{s}\right] d x d t \\
& \quad+\int_{\left\{\left|u_{n}\right| \leq l\right\}} a\left(x, t, u_{n}, \nabla u_{n}\right)\left[\nabla v_{k} \cdot \chi_{k}^{s}-\nabla u \cdot \chi^{s}\right] d x d t \\
& \quad-\int_{\left\{\left|u_{n}\right| \leq l\right\}} a\left(x, t, u_{n}, \nabla v_{k} \cdot \chi_{k}^{s}\right)\left[\nabla u_{n}-\nabla v_{k} \cdot \chi_{k}^{s}\right] d x d t \\
& :=I_{1}+I_{2}+I_{3} .
\end{aligned}
$$

We shall go to the limit, first as $n \rightarrow \infty$ and next as $k \rightarrow \infty$ and finally as $s \rightarrow \infty$, in all integrals $I_{i}$, for $i=1,2,3$.

Since $\chi_{\left\{\left|u_{n}\right| \leq l\right\}} a\left(x, t, u_{n}, \nabla v_{k} \cdot \chi_{k}^{s}\right) \rightarrow \chi_{\{|u| \leq l\}} a\left(x, t, u, \nabla v_{k} \cdot \chi_{k}^{s}\right)$ strongly in $\left(E_{\bar{M}}(Q)\right)^{N}$, by (4) and the fact that $u_{n} \rightarrow u$ a.e. in $Q$ we deduce that

$$
I_{1} \rightarrow \int_{\{|u| \leq l\} \cap\{|\nabla u| \geq s\}} a(x, t, u, 0) \nabla u d x d t \quad \text { as } n \rightarrow \infty,
$$

which clearly tends to zero as $s \rightarrow \infty$.

Observe that $I_{2}$ tends to

$$
\int_{\{|u| \leq l\}} h\left[\nabla v_{k} \cdot \chi_{k}^{s}-\nabla u \cdot \chi^{s}\right] d x d t \quad \text { as } n \rightarrow \infty
$$

which tends to 0 as $k \rightarrow \infty$ since $\nabla v_{k} \cdot \chi_{k}^{s}-\nabla u \cdot \chi^{s} \rightarrow 0$ strongly in $\left(E_{M}(Q)\right)^{N}$.

For the third term $I_{3}$, since $\nabla u_{n} \rightarrow \nabla u$ in $\left(L_{M}(Q)\right)^{N}$, we have

$$
I_{3} \rightarrow-\int_{\{|u| \leq l\}} a\left(x, t, u, \nabla v_{k} \cdot \chi_{k}^{s}\right)\left[\nabla u-\nabla v_{k} \cdot \chi_{k}^{s}\right] d x d t \quad \text { as } n \rightarrow \infty
$$

since $\chi_{\{|u| \leq l\}} a\left(x, t, u, \nabla v_{k} \cdot \chi_{k}^{s}\right) \rightarrow \chi_{\{|u| \leq l\}} a\left(x, t, u, \nabla u \cdot \chi^{s}\right)$ strongly in $\left(E_{\bar{M}}(Q)\right)^{N}$ as $k \rightarrow \infty$ by Lebesgue's theorem, the above tends to

$$
-\int_{\{|u| \leq l\} \cap\{|\nabla u| \geq s\}} a(x, t, u, 0) \nabla u d x d t
$$

as $k \rightarrow \infty$, which clearly tends to zero as $s \rightarrow \infty$.

We have thus proved that

$$
\begin{aligned}
& \int_{\left\{\left|u_{n}\right| \leq l\right\}}\left[a\left(x, t, u_{n}, \nabla u_{n}\right)-a\left(x, t, u_{n}, \nabla u \cdot \chi^{s}\right)\right]\left[\nabla u_{n}-\nabla u \cdot \chi^{s}\right] d x d t \\
= & \int_{\left\{\left|u_{n}\right| \leq l\right\}}\left[a\left(x, t, u_{n}, \nabla u_{n}\right)-a\left(x, t, u_{n}, \nabla v_{k} \cdot \chi_{k}^{s}\right)\right]\left[\nabla u_{n}-\nabla v_{k} \cdot \chi_{k}^{s}\right] d x d t+\varepsilon(n, k, s),
\end{aligned}
$$

where $\varepsilon(n, k, s)$ denotes quantities (possibly different) depending on $l$ such that

$$
\lim _{s \rightarrow \infty} \lim _{k \rightarrow \infty} \lim _{n \rightarrow \infty} \varepsilon(n, k, s)=0 .
$$


For all $s \geq r>0$ and all $l \geq \bar{l}$, we have

$$
\begin{aligned}
& \text { (14) } 0 \leq \int_{\left\{\left|u_{n}\right| \leq \bar{l},|\nabla u| \leq r\right\}}\left[a\left(x, t, u_{n}, \nabla u_{n}\right)-a\left(x, t, u_{n}, \nabla u\right)\right]\left[\nabla u_{n}-\nabla u\right] d x d t \\
& \leq \int_{\left\{\left|u_{n}\right| \leq l,|\nabla u| \leq s\right\}}\left[a\left(x, t, u_{n}, \nabla u_{n}\right)-a\left(x, t, u_{n}, \nabla u\right)\right]\left[\nabla u_{n}-\nabla u\right] d x d t \\
& \leq \int_{\left\{\left|u_{n}\right| \leq l\right\}}\left[a\left(x, t, u_{n}, \nabla u_{n}\right)-a\left(x, t, u_{n}, \nabla u \cdot \chi^{s}\right)\right]\left[\nabla u_{n}-\nabla u \cdot \chi^{s}\right] d x d t \\
& =\int_{\left\{\left|u_{n}\right| \leq l\right\}}\left[a\left(x, t, u_{n}, \nabla u_{n}\right)-a\left(x, t, u_{n}, \nabla v_{k} \cdot \chi_{k}^{s}\right)\right]\left[\nabla u_{n}-\nabla v_{k} \cdot \chi_{k}^{s}\right] d x d t \\
& +\varepsilon(n, k, s) \\
& =-\int_{\left\{\left|u_{n}\right| \leq l\right\}} a\left(x, t, u_{n}, \nabla v_{k} \cdot \chi_{k}^{s}\right)\left[\nabla u_{n}-\nabla v_{k} \cdot \chi_{k}^{s}\right] d x d t \\
& +\int_{Q}\left(a\left(x, t, u_{n}, \nabla u_{n}\right)\left[\nabla u_{n}-\nabla v_{k}\right]+a_{0}\left(x, t, u_{n}, \nabla u_{n}\right)\left(u_{n}-v_{k}\right)\right) d x d t \\
& -\left(\int_{\left\{\left|u_{n}\right|>l\right\}} a\left(x, t, u_{n}, \nabla u_{n}\right)\left[\nabla u_{n}-\nabla v_{k}\right] d x d t\right. \\
& \left.+\int_{Q} a_{0}\left(x, t, u_{n}, \nabla u_{n}\right)\left(u_{n}-v_{k}\right) d x d t\right) \\
& +\int_{\left\{\left|u_{n}\right| \leq l\right\} \cap\left\{\left|\nabla v_{k}\right|>s\right\}} a\left(x, t, u_{n}, \nabla u_{n}\right) \nabla v_{k} d x d t+\varepsilon(n, k, s) \\
& :=J_{1}+J_{2}+J_{3}+J_{4}+\varepsilon(n, k, s) \text {. }
\end{aligned}
$$

We shall take the limsup first over $n$ and next over $k$ and finally over $s$ in all integrals of the right hand side.

Remark that, by (13),

$$
\limsup _{k \rightarrow \infty} \limsup _{n \rightarrow \infty} J_{2} \leq 0 .
$$

Just as for $I_{1}$ above, it is easy to see that

$$
\lim _{s \rightarrow \infty} \limsup _{k \rightarrow \infty} \limsup _{n \rightarrow \infty} J_{1}=0 .
$$

The third term reads

$$
\begin{aligned}
J_{3}= & -\int_{\left\{\left|u_{n}\right|>l\right\}}\left[a\left(x, t, u_{n}, \nabla u_{n}\right)\left[\nabla u_{n}-\nabla v_{k}\right]+a_{0}\left(x, t, u_{n}, \nabla u_{n}\right)\left(u_{n}-v_{k}\right)\right] d x d t \\
& -\int_{\left\{\left|u_{n}\right| \leq l\right\}} a_{0}\left(x, t, u_{n}, \nabla u_{n}\right)\left(u_{n}-v_{k}\right) d x d t
\end{aligned}
$$


and, by using (7),

$$
\begin{aligned}
J_{3} \leq & \int_{\left\{\left|u_{n}\right|>l\right\}}\left[a\left(x, t, u_{n}, \nabla u_{n}\right) \nabla v_{k}+a_{0}\left(x, t, u_{n}, \nabla u_{n}\right) v_{k}\right] d x d t \\
& +\int_{\left\{\left|u_{n}\right|>l\right\}} d(x, t) d x d t-\int_{\left\{\left|u_{n}\right| \leq l\right\}} a_{0}\left(x, t, u_{n}, \nabla u_{n}\right)\left(u_{n}-v_{k}\right) d x d t
\end{aligned}
$$

which gives

$$
\begin{aligned}
\limsup _{n \rightarrow \infty} J_{3} \leq & \int_{\{|u| \geq l\}}\left(h \nabla v_{k}+h_{0} v_{k}\right) d x d t+\int_{\{|u| \geq l\}} d(x, t) d x d t \\
& -\int_{\{|u| \leq l\}} h_{0}\left(u-v_{k}\right) d x d t,
\end{aligned}
$$

where we have used the strong convergence of $\chi_{\left\{\left|u_{n}\right|>l\right\}}\left|\nabla v_{k}\right|$ and $\chi_{\left\{\left|u_{n}\right|>l\right\}} v_{k}$ and $\chi_{\left\{\left|u_{n}\right| \leq l\right\}} u_{n}$ in $E_{M}(Q)$ as $n \rightarrow \infty$. This implies that

$$
\limsup _{k \rightarrow \infty} \limsup _{n \rightarrow \infty} J_{3} \leq \int_{\{|u| \geq l\}}\left(h \nabla u+h_{0} u\right) d x d t+\int_{\{|u| \geq l\}} d(x, t) d x d t
$$

since $v_{k} \rightarrow u$ in $W_{0}^{1, x} L_{M}(Q)$ for the modular convergence.

For $J_{4}$, we have

$$
\lim _{n \rightarrow \infty} J_{4}=\int_{\{|u| \leq l\} \cap\left\{\left|\nabla v_{k}\right|>s\right\}} h \nabla v_{k} d x d t
$$

since $\chi_{\left\{\left|u_{n}\right| \leq l,\left|\nabla v_{k}\right|>s\right\}} \nabla v_{k} \rightarrow \chi_{\left\{|u| \leq l,\left|\nabla v_{k}\right|>s\right\}} \nabla v_{k}$ strongly in $\left(E_{M}(Q)\right)^{N}$ as $n \rightarrow \infty$. This implies that

$$
\lim _{k \rightarrow \infty} \lim _{n \rightarrow \infty} J_{4}=\int_{\{|u| \leq l\} \cap\{|\nabla u|>s\}} h \nabla u d x d t \leq \int_{\{|\nabla u| \geq s\}}|h \nabla u| d x d t
$$

and thus

$$
\limsup _{s \rightarrow \infty} \lim _{k \rightarrow \infty} \lim _{n \rightarrow \infty} J_{4} \leq 0 .
$$

Combining these estimates with (14) and taking the limsup first over $n$, then over $k$ and next over $s$, we deduce that

$$
\begin{aligned}
0 & \leq \limsup _{n \rightarrow \infty} \int_{\left\{\left|u_{n}\right| \leq \bar{l},|\nabla u| \leq r\right\}}\left[a\left(x, t, u_{n}, \nabla u_{n}\right)-a\left(x, t, u_{n}, \nabla u\right)\right]\left[\nabla u_{n}-\nabla u\right] d x d t \\
& \leq \int_{\{|u| \geq l\}}\left(h \nabla u+h_{0} u+d(x, t)\right) d x d t,
\end{aligned}
$$

in which we can let $l \rightarrow \infty$ to get

$$
\lim _{n \rightarrow \infty} \int_{\left\{\left|u_{n}\right| \leq \bar{l},|\nabla u| \leq r\right\}}\left[a\left(x, t, u_{n}, \nabla u_{n}\right)-a\left(x, t, u_{n}, \nabla u\right)\right]\left[\nabla u_{n}-\nabla u\right] d x d t=0
$$


and thus, as in the elliptic case (see [2]), we deduce that, for a subsequence still denoted by $u_{n}$,

$$
\nabla u_{n} \rightarrow \nabla u \quad \text { a.e. in } Q .
$$

This implies that $h=a(x, t, u, \nabla u)$ and $h_{0}=a_{0}(x, t, u, \nabla u)$. Therefore, for all $\varphi \in C^{1}([0, T], \mathcal{D}(\Omega))$ we get

$$
\begin{aligned}
-\int_{Q} u \frac{\partial \varphi}{\partial t} d x d t+\left[\int_{\Omega} u(t) \varphi(t) d x\right]_{0}^{T} & +\int_{Q} a(x, t, u, \nabla u) \nabla \varphi d x d t \\
& +\int_{Q} a_{0}(x, t, u, \nabla u) \varphi d x d t=\langle f, \varphi\rangle .
\end{aligned}
$$

STEP 3. Regularity of the solution. Note that we may choose $v_{k}$ such that

$$
\lim _{n \rightarrow \infty} \int_{Q_{\tau}} \frac{\partial v_{k}}{\partial t}\left(v_{k}-u_{n}\right) d x d t \leq \varepsilon_{k}
$$

uniformly in $\tau \in[0, T]$, where $\varepsilon_{k} \rightarrow 0$ as $k \rightarrow \infty$.

For all $k$ and all $\tau$ in $(0, T)$, from (10) we have

$\lim _{n \rightarrow \infty} \int_{Q_{\tau}} \frac{\partial u_{n}}{\partial t} v_{k} d x d t+\int_{Q_{\tau}} a(x, t, u, \nabla u) \nabla v_{k} d x d t+\int_{Q_{\tau}} a_{0}(x, t, u, \nabla u) v_{k} d x d t$ $=\left\langle f, v_{k}\right\rangle_{Q_{\tau}}$,

which implies, by using Fatou's lemma,

$$
\begin{aligned}
\limsup _{n \rightarrow \infty} \int_{Q_{\tau}} \frac{\partial u_{n}}{\partial t}\left(u_{n}-v_{k}\right) d x d t \\
=-\liminf _{n \rightarrow \infty} \int_{Q_{\tau}}\left(a\left(x, t, u_{n}, \nabla u_{n}\right) \nabla u_{n}+a_{0}\left(x, t, u_{n}, \nabla u_{n}\right) u_{n}\right) d x d t \\
\quad+\int_{Q_{\tau}}\left(a(x, t, u, \nabla u) \nabla v_{k}+a_{0}(x, t, u, \nabla u) v_{k}\right) d x d t \\
\quad+\lim _{n \rightarrow \infty} \int_{Q_{\tau}} f_{n}\left(u_{n}-v_{k}\right) d x d t \\
\leq-\int_{Q_{\tau}}\left(a(x, t, u, \nabla u) \nabla u+a_{0}(x, t, u, \nabla u) u\right) d x d t \\
\quad+\int_{Q_{\tau}}\left(a(x, t, u, \nabla u) \nabla v_{k}+a_{0}(x, t, u, \nabla u) v_{k}\right) d x d t+\left\langle f, u-v_{k}\right\rangle_{Q_{\tau}}
\end{aligned}
$$

and hence

$$
\limsup _{n \rightarrow \infty} \int_{Q_{\tau}}\left(\frac{\partial u_{n}}{\partial t}-\frac{\partial v_{k}}{\partial t}\right)\left(u_{n}-v_{k}\right) d x d t \leq \varepsilon_{k}+\varepsilon_{k}^{\prime}
$$


uniformly in $\tau \in[0, T]$. Since

$$
\begin{aligned}
\limsup _{n \rightarrow \infty} \int_{Q_{\tau}}\left(\frac{\partial u_{n}}{\partial t}-\frac{\partial v_{k}}{\partial t}\right)\left(u_{n}-v_{k}\right) d x d t & =\limsup _{n \rightarrow \infty}\left[\frac{1}{2} \int_{\Omega}\left(u_{n}-v_{k}\right)^{2} d x\right]_{0}^{\tau} \\
& =\frac{1}{2} \limsup _{n \rightarrow \infty} \int_{\Omega}\left(u_{n}(\tau)-v_{k}(\tau)\right)^{2} d x,
\end{aligned}
$$

we deduce the inequality

$$
\limsup _{n \rightarrow \infty} \int_{\Omega}\left(u_{n}(\tau)-v_{k}(\tau)\right)^{2} d x \leq 2 \varepsilon_{k}+2 \varepsilon_{k}^{\prime}
$$

implying that $\left(v_{k}\right)$ is a Cauchy sequence in $C\left([0, T], L^{2}(\Omega)\right)$ and that $u \in$ $C\left([0, T], L^{2}(\Omega)\right)$.

Corollary 1. The function $u$ can be used as a testing function, i.e.

$$
\begin{array}{r}
\frac{1}{2}\left[\int_{\Omega}(u(t))^{2} d x\right]_{0}^{\tau}+\int_{Q_{\tau}} a(x, t, u, \nabla u) \nabla u d x d t+\int_{Q_{\tau}} a_{0}(x, t, u, \nabla u) u d x d t \\
=\langle f, u\rangle_{Q_{\tau}} .
\end{array}
$$

Proof. As in [15], by using Fatou's lemma we have

$$
\begin{aligned}
0 \leq & \lim _{k \rightarrow \infty} \lim _{n \rightarrow \infty} \int_{Q_{\tau}}\left(a\left(x, t, u_{n}, \nabla u_{n}\right)\left(\nabla u_{n}-\nabla v_{k}\right)\right. \\
& \left.+a_{0}\left(x, t, u_{n}, \nabla u_{n}\right)\left(u_{n}-v_{k}\right)\right) d x d t \\
= & \lim _{k \rightarrow \infty} \lim _{n \rightarrow \infty} \int_{Q_{\tau}} \frac{\partial u_{n}}{\partial t}\left(v_{k}-u_{n}\right) d x d t+\lim _{k \rightarrow \infty} \lim _{n \rightarrow \infty}\left\langle f_{n}, u_{n}-v_{k}\right\rangle_{Q_{\tau}} \\
\leq & \frac{1}{2} \lim _{k \rightarrow \infty} \limsup _{n \rightarrow \infty}\left[\int_{\Omega}-\left(u_{n}(t)-v_{k}(t)\right)^{2} d x\right]_{0}^{\tau} \\
& +\lim _{k \rightarrow \infty} \limsup _{n \rightarrow \infty} \int_{Q_{\tau}} \frac{\partial v_{k}}{\partial t}\left(v_{k}-u_{n}\right) d x d t \leq 0 .
\end{aligned}
$$

This implies that

$$
\begin{aligned}
\lim _{n \rightarrow \infty} \int_{Q_{\tau}}\left(a\left(x, t, u_{n}, \nabla u_{n}\right)\right. & \left.\nabla u_{n}+a_{0}\left(x, t, u_{n}, \nabla u_{n}\right) u_{n}\right) d x d t \\
& =\int_{Q_{\tau}}\left(a(x, t, u, \nabla u) \nabla u+a_{0}(x, t, u, \nabla u) u\right) d x d t
\end{aligned}
$$

and

$$
\frac{1}{2} \lim _{n \rightarrow \infty}\left\|u_{n}(t)-v_{k}(t)\right\|_{L^{2}(\Omega)}^{2} \leq \varepsilon_{k}+\varepsilon_{k}^{\prime} .
$$

Since $v_{k}(\tau) \rightarrow u(\tau)$ in $L^{2}(\Omega)$, we also have $u_{n}(\tau) \rightarrow u(\tau)$ in $L^{2}(\Omega)$ and then

$$
\lim _{n \rightarrow \infty} \int_{Q_{\tau}} \frac{\partial u_{n}}{\partial t} u_{n} d x d t=\frac{1}{2}\left[\int_{\Omega}(u(t))^{2} d x\right]_{0}^{\tau} .
$$


Therefore, it is easy to pass to the limit in (10) with $\varphi=u_{n}$ to get the result.

\section{References}

[1] R. Adams, Sobolev Spaces, Academic Press, New York, 1975.

[2] A. Benkirane and A. Elmahi, Almost everywhere convergence of the gradients of solutions to elliptic equations in Orlicz spaces and application, Nonlinear Anal. 28 (1997), 1769-1784.

[3] L. Boccardo and F. Murat, Strongly nonlinear Cauchy problems with gradient dependent lower order nonlinearity, in: Pitman Res. Notes Math. Ser. 208, Longman, 1989, 247-254.

[4] —, - Almost everywhere convergence of the gradients, Nonlinear Anal. 19 (1992), 581-597.

[5] H. Brézis and F. E. Browder, Strongly nonlinear parabolic initial boundary value problems, Proc. Nat. Acad. Sci. U.S.A. 76 (1979), 38-40.

[6] T. Donaldson, Inhomogeneous Orlicz-Sobolev spaces and nonlinear parabolic initialboundary value problems, J. Differential Equations 16 (1974), 201-256.

[7] A. Elmahi, Strongly nonlinear parabolic initial-boundary value problems in Orlicz spaces, Electron. J. Differ. Equ. Conf. 9 (2002), 203-220.

[8] A. Elmahi and D. Meskine, Strongly nonlinear parabolic equations with natural growth terms in Orlicz spaces, Nonlinear Anal. 60 (2005), 1-35.

[9] J.-P. Gossez, Nonlinear elliptic boundary value problems for equations with rapidly (or slowly) increasing coefficients, Trans. Amer. Math. Soc. 190 (1974), 163-205.

[10] - Some approximation properties in Orlicz-Sobolev spaces, Studia Math. 74 (1982), $17-24$.

[11] J.-P. Gossez and V. Mustonen, Variational inequalities in Orlicz-Sobolev spaces, Nonlinear Anal. 11 (1987), 379-392.

[12] M. Krasnosel'skiı̌ and Ya. Rutickiǔ, Convex Functions and Orlicz Spaces, Noordhoff, Groningen, 1969.

[13] A. Kufner, O. John and S. Fučík, Function Spaces, Academia, Prague, 1977.

[14] R. Landes, On the existence of weak solutions for quasilinear parabolic initialboundary value problems, Proc. Roy. Soc. Edinburgh Sect. A 89 (1981), 217-137.

[15] R. Landes and V. Mustonen, A strongly nonlinear parabolic initial-boundary value problem, Ark. Mat. 25 (1987), 29-40.

[16] J.-L. Lions, Quelques méthodes de résolution des problèmes aux limites non linéaires, Dunod et Gauthier-Villars, 1969.

[17] C. B. Morrey Jr., Multiple Integrals in the Calculus of Variations, Springer, Berlin, 1969.

[18] J. Robert, Inéquations variationnelles paraboliques fortement non linéaires, J. Math. Pures Appl. 53 (1974), 299-321.

A. Elmahi

C.P.R., B.P. 49

Fès, Morocco

E-mail: elmahi_abdelhak@yahoo.fr
D. Meskine

Département de Mathématiques et Informatique Faculté des Sciences Dhar Mahrazr, B.P. 1796

Atlas, Fès, Morocco E-mail: meskinedriss@hotmail.com 